\title{
Compressive Sensing Based Separation of LFM Signals
}

\author{
Irena Orović, Srdjan Stanković and Ljubiša Stanković \\ University of Montenegro, Faculty of Electrical Engineering, 81000 Podgorica, Montenegro \\ irenao@ac.me
}

\begin{abstract}
A compressive sensing approach for separation of linear frequency modulated signals from non-stationary disturbance is proposed. The linear time-frequency representation is achieved using the Local Polynomial Fourier Transform (LPFT), which allows revealing data local behavior. Based on the LPFT, the frequency-chirp rate domain is used to achieve sparse signal representation. Then the LPFT is combined with the L-statistics to collect only the time-frequency points belonging to the desired signal, while the points belonging to overlapping regions and disturbance are deemed inappropriate and omitted from observations. The relationship between the measurement and sparsity domain is established in order to use the compressive sensing concept and to completely recover the desired signal. The theory is proven on examples.
\end{abstract}

Keywords - time-frequency analysis, compressive sensing, Lestimation, signal separation

\section{INTRODUCTION}

Signals in real applications, such as in radars and communications, are often disturbed by different kinds of interferences that are produced by different sources and different physical processes. The components of interest (desired components) could be seriously interrupted by impulse noise, clutters, frequency hopping jammer, etc [1]-[3]. Therefore, if these components highly overlap in time and frequency, signal separation and desired signal recovery can be hardly accomplished through conventional methods and filtering techniques [4],[5]. Moreover, the time-frequency (TF) content of desired and undesired signals can reside over common TF regions and thus it is difficult to provide the separation in the TF domain using TF masking and synthesis methods. Also, the disturbance can be much stronger than the desired signal in the overlapping regions. Hence, we need to exclude all observations containing the disturbances. For this purpose, we use the L-statistics to isolate the TF points belonging only to the signal of interest [6]-[8]. Such an approach can be efficient only if the desired components have stationary nature [10]. In that sense, when dealing with timevarying frequency content as in the case of linear frequency modulated signals (LFM), we propose to use the Local Polynomial Fourier Transform (LPFT), which provides the TF representation of demodulated signal [11]. As a consequence of applying the L-statistics, we deal with missing observations in the TF domain. The theory that considers reconstruction of signals, with sparse representations in certain transform domain, using an incomplete set of samples is known as Compressive sensing (CS) [12]-[18]. Although, in the considered application, the missing samples are not due to Nyquist sampling relaxation as in the case of standard CS concept, we might benefit from the CS reconstruction algorithms. Unlike the standard CS formulations and reconstructions [16], here the observations are made in the TF domain instead of the time domain. Thus, the problem is observed as a CS application aiming at recovery of narrowband signals in interference, using $\ell_{1}$ reconstruction algorithms. Unlike the other existing methods, the proposed one provides efficient results with preserved amplitudes and phases.

The paper is organized as follows. The theoretical background on the L-statistics and time-frequency analysis is given in Section 2. The LFM components separation based on the LPFT, the L-estimation and the CS is proposed in Section 3. The experimental results are presented in Section 4, while the concluding remarks are given in Section 5.

\section{THEORETICAL BACKGROUND - L-ESTIMATION AND TIME-FREQUENCY ANALYSIS}

\section{A. Problem formulation}

Consider the case of signal corrupted by impulse noise or certain disturbances (impurity components) that impede the analysis of useful components. For example, the narrowband signals in communications may be disturbed by a frequency hopping jammer that is of shorter duration than the considered time-interval, but may also be overlapping with narrowband signals within same intervals. The problem formulation can be stated as follows. Consider a composite signal:

$$
x(n)=f(n)+\varepsilon(n),
$$

where $f(n)$ represents useful signal part, while $\varepsilon(n)$ represents the impurity components. The discrete Fourier transform (DFT) of signal $x(n)$ can, therefore, be defined as:

$$
X(k)=F(k)+\mathrm{E}(k),
$$

where $F(k) \neq 0$ for $k \in\left\{k_{1}, k_{2}, \ldots k_{K}\right\}, K<<N$ ( $N$ is the number of time samples). Furthermore, we assume that certain frequency components in $\mathrm{E}(k)$ could be much stronger than their counterparts in $F(k)$ : 


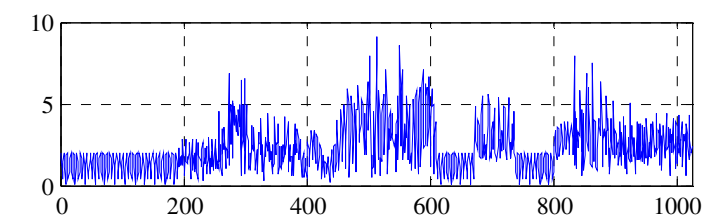

a)

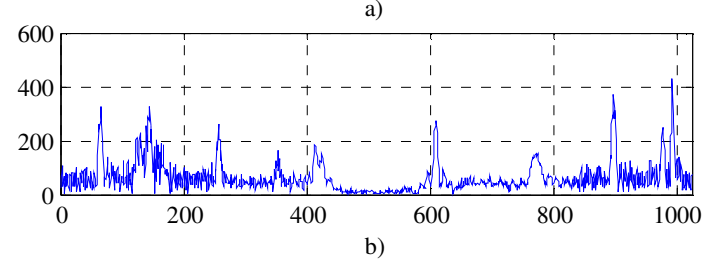

Figure 1. a) real part of signal in time domain, b) DFT of signal

$$
\left|E\left(k_{i}\right)\right| \gg\left|F\left(k_{i}\right)\right|, i=1,2, \ldots, K .
$$

An illustration for the DFT of chirp signal with several impulsive components is shown in Fig. 1. When observing the signals in the frequency domain, we can hardly distinguish the type, source and duration of components, and thus it is usually difficult, if not impossible, to separate different components. This is the reason that we often prompt towards the TF analysis of such signals.

\section{B. L-estimation and time-frequency analysis}

The short-time Fourier transform (STFT) is the simplest time-frequency representation calculated using a rectangular window of the width $M$ as follows:

$$
\operatorname{STFT}(n, k)=\sum_{m=0}^{M-1} x(n+m) e^{-j 2 \pi m k / M}
$$

The values of the STFT are sorted for each frequency $k$ along the time axis $n$. By removing several strongest values of the sorted STFT, for each frequency, we eliminate most or all of the disturbance components. Summing the rest of the STFT values over time will result in the spectrum of the desired signal part. For a given frequency $k$, let us observe one STFT column with $M$ elements:

$$
S_{k}(m)=\operatorname{sort}\{\operatorname{STFT}(m, k), m=0, \ldots, M-1\},
$$

such that $\left|S_{k}(0)\right| \leq\left|S_{k}(1)\right| \leq \ldots \leq\left|S_{k}(M-1)\right|$. Since the addition is a commutative operation, using the entire data set yields [8]:

$$
\sum_{m=0}^{M-1} \operatorname{STFT}(m, k)=\sum_{m=0}^{M-1} S_{k}(m)=S(k) .
$$

If we discard $Q$ highest values and $P$ smallest values of $S_{k}$ for each $k$, we will produce an estimate of $S(k)$, denoted by $S_{L}(k)$, as follows:

$$
S_{L}(k)=\sum_{m=P}^{M-Q} S_{k}(m)
$$

In the data analysis, this approach, based on elimination of a certain part of data, before analyzing the rest of the data, is known as the L-statistics. The L-statistics has been already used for realizations of signal transforms and representations when signal is heavily corrupted by impulse noise [7]-[10]. If there is only a disturbance at certain frequency in STFT plane, then omitting highest values along that frequency would eliminate the interference. If at certain frequency there is a contribution from both the interference and the desired components, the highest values would correspond to overlapping points. Finally, when the interference and desired components are of opposite phases, it will produce low amplitude at the overlapping point. For that reason it is necessary to remove some of the lowest values, as well. Consequently, the missing samples appear as a consequence of attempting to separate desired from undesired components.

\section{CS BASED COMPONENTS SEPARATION}

The concept of compressive sensing reconstruction can be used to recover narrowband signals when contaminated with different impulsive disturbances. In this case, the desired sparse representation can be achieved using the Fourier transform. Furthermore, large number of signals, especially the sinusoids, are both locally and globally sparse in the timefrequency domain. The local behavior of sinusoids is revealed by taking the STFT [10]. However, when dealing with LFM signals or some other known IF laws, we propose to use the LPFT. In this way, we demodulate components of interest and the scenario is again reduced to the sparse sinusoidal components. The time-frequency regions corresponding to the non-stationary disturbance over all windows are identified and removed from consideration, using the previously presented L-estimation approach. Based on the set of remaining TF points, the CS reconstruction methods will be employed to provide the exact recovery of sparse signal.

Let us observe the signal $\mathbf{x}$ which is made of time-varying LFM signal $\mathbf{f}$ and a set of strong non-stationary disturbances $\boldsymbol{\varepsilon}$. In the considered case, it is obvious that prior to the Lestimation approach which aims to remove all disturbances, we need to stationarize the desired components in f. Hence, the idea is to use the LPFT, which can be defined as:

$$
\operatorname{LPFT}(n, k)=\sum_{m=0}^{M-1} x(m) w(m-n) e^{-j 2 \pi\left(m k+\alpha m^{2}\right) / M},
$$

where $w$ is a window function (without loss of generality the rectangular window can be assumed). The term $\exp \left(-j \alpha m^{2}\right)$ is used to compensate the linear frequency modulated part of the signal, or in other words to demodulate the signal. The LFM signals are sparse in the frequency-chirp rate domain. Like the signal frequency, the parameter $\alpha$, i.e., the chirp rate is not known in advance, but we can calculate LPFT for all values from a certain set $\left[\alpha_{\min }, \alpha_{\max }\right]$. The exact value of $\alpha$ can be estimated as the value which provides the highest concentration (the highest peak when summing up the LPFT values along time axes). Alternatively, we can estimate $\alpha$ as a value that produces representation which at certain frequency has $M$ non-zero values higher than a threshold. Here, it is also important to emphasize that the components within the 
multicomponent LFM signal may have different chirp rates. The LPFT in the matrix form can be written as:

$$
\operatorname{LPFT}_{M}(n)=\mathcal{F}_{M} \mathbf{z}(n),
$$

where $\mathbf{L P F T}_{M}(n)$ and $\mathbf{z}(n)\left(\mathbf{x}(n)\right.$ multiplied by $\left.\exp \left(-j \alpha n^{2}\right)\right)$ are vectors in the form:

$$
\begin{gathered}
\operatorname{LPFT}_{M}(n)=[\operatorname{LPFT}(n, 0), \ldots, \operatorname{LPFT}(n, M-1)]^{T}, \\
\mathbf{z}(n)=[z(n), \mathrm{z}(n+1), \ldots, z(n+M-1)]^{T},
\end{gathered}
$$

while $\mathcal{F}_{M}$ is $M \times M$ DFT matrix with elements:

$$
\begin{gathered}
\mathcal{F}(m, k)=e^{-j 2 \pi k m / M}, \\
m=0, \ldots, M-1, \quad k=0, \ldots, M-1
\end{gathered} .
$$

We assume that non-overlapping windows are used for LPFT calculation. Hence, after calculating all LPFT vectors, we can combine them in a single equation:

$$
\mathbf{L P F T}=\mathcal{F}_{M, N} \mathbf{z},
$$

such that vector LPFT is composed of vectors: $\mathbf{L P F T}_{\mathrm{M}}(0)$, $\mathbf{L P F T}_{M}(M), \ldots, \mathbf{L P F T}_{M}(N-M)$. The matrix $\mathcal{F}_{M, N}$ of size $N \times N$ is obtained as a Kronecker product:

$$
\mathcal{F}_{M, N}=\mathbf{I}_{N / M} \otimes \mathcal{F}_{M},
$$

where $\mathbf{I}_{N / M}$ denotes the identity matrix of size $(N / M) \times(N / M)$. Now, if we express $\mathbf{z}$ using its Fourier transform vector $\mathbf{Z}$, we may write:

$$
\mathbf{L P F T}=\mathcal{F}_{M, N} \mathcal{F}_{N}^{-1} \mathbf{Z},
$$

where $\mathcal{F}_{N}^{-1}$ is the inverse DFT matrix of size $N \times N$, or:

$$
\mathbf{L P F T}=\boldsymbol{\Psi Z} .
$$

with $\boldsymbol{\Psi}=\mathcal{F}_{M, N} \mathcal{F}_{N}^{-1}$. The L-estimation is now applied to the LPFT as follows. For each frequency $k$, a vector of LPFT in time is formed as:

$$
\left[\mathbf{S}_{\mathbf{k}}(m), \mathbf{p}\right]=\operatorname{sort}\{\operatorname{LPFT}(m, k), m=0, \ldots, M-1\},
$$

where $\mathbf{p}$ denotes the vector of positions in the original (nonsorted vector). Then we have:

$$
\begin{gathered}
\mathbf{L P F T}_{k}^{C S}=\left\{S_{k}(m) \mid m=P, P+1, P+2 \ldots, M-Q\right\}, \\
\mathbf{p}_{k}^{C S}=\mathbf{p}\{P, P+1, P+2, \ldots, M-Q\}
\end{gathered},
$$

where $\mathbf{p}_{k}^{C S}$ denotes positions of remaining CS coefficients. Finally, when considering all frequencies $k$, the vector of all available LPFT values is denoted as LPFT $^{\mathrm{CS}}$. The corresponding CS matrix $\mathbf{A}$ is formed by omitting the rows in $\boldsymbol{\Psi}$ that correspond to the removed positions in LPFT $^{\mathbf{C S}}$ vector. Each row corresponds to one time and frequency point $(n, k)$. In order to reconstruct the original sparse stationary signal, the CS minimization problem can be defined as follows:

$$
\min \|\mathbf{Z}\|_{\ell_{1}} \text { subject to } \mathbf{L P F T}^{\mathrm{CS}}=\mathbf{A} \cdot \mathbf{Z} \text {. }
$$

The reconstructed DFT vector $\mathbf{Z}$ is used to obtain the time domain signal $z(n)$, which is then re-modulated to LFM signal by multiplication with the factor $\exp \left(j \alpha n^{2}\right)$. The same results can be obtained by using the fractional Fourier transform instead of the LPFT, with the rotation parameter instead of chirp rate.

\section{EXPERIMENTAL RESULTS}

Consider the sparse signal in the form:

$f(t)=e^{\left(-j 640 \pi n^{2} / N+j 256 \pi n / N\right)}+e^{\left(-j 640 \pi n^{2} / N-j 128 \pi n / N\right)}$ corrupted by the nonstationary disturbances. The disturbances are of the form of short duration modulated signals (some of them are at the same frequencies as the stationary sinusoids):

$$
\varepsilon(n)=\sum_{i=1}^{K} A_{i} e^{j a_{i} n} e^{-\left(n-n_{i}\right)^{2} / b_{i}^{2}},
$$

Different amplitudes are assumed for 19 components all in the range between $A i=1$ and $A i=4$, with different durations defined by $b i$. Some of the disturbance terms appear at the same frequencies as the desired components. The STFT is calculated for $N=1024$ and $M=32$. The LPFT is calculated for a set of values $\alpha$ between $\alpha_{\min }=-1024$ to $\alpha_{\max }=1024$. The STFT of original disturbed data is presented in Fig. 2.a. The LPFT of demodulated signal is shown in Fig 2.b. The sorted LPFT values are shown in Fig 2.c. The L-statistics is applied to the sorted LPFT: $50 \%$ of the largest values are removed along with $10 \%$ of the smallest values. The reconstructed sinusoids in the STFT domain are shown in Fig 2.d.

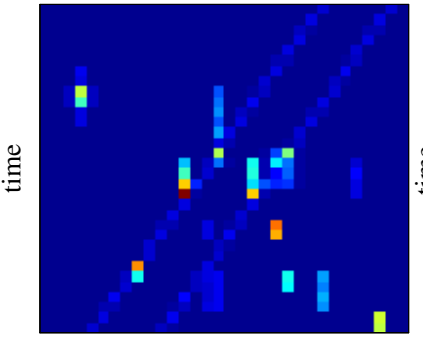

frequency

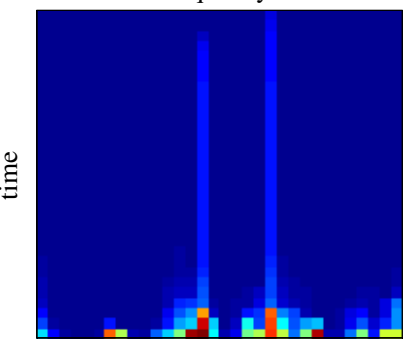

frequency

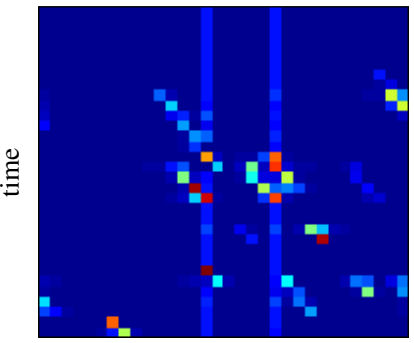

frequency

frequency

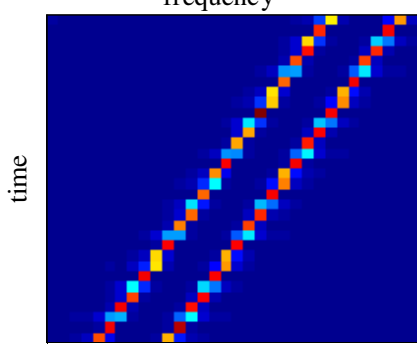

Figure 2. a) STFT of original signal, b) STFT of demodulated signal, c) sorted STFT, d) STFT of reconstructed re-modulated signal 

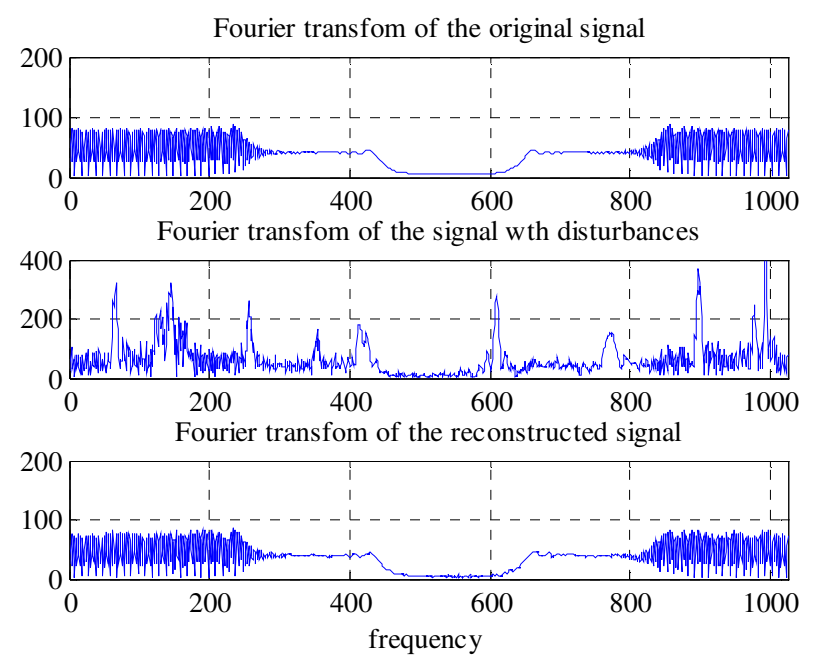

Figure 3. FT of original signal, noisy signal and reconstructed one
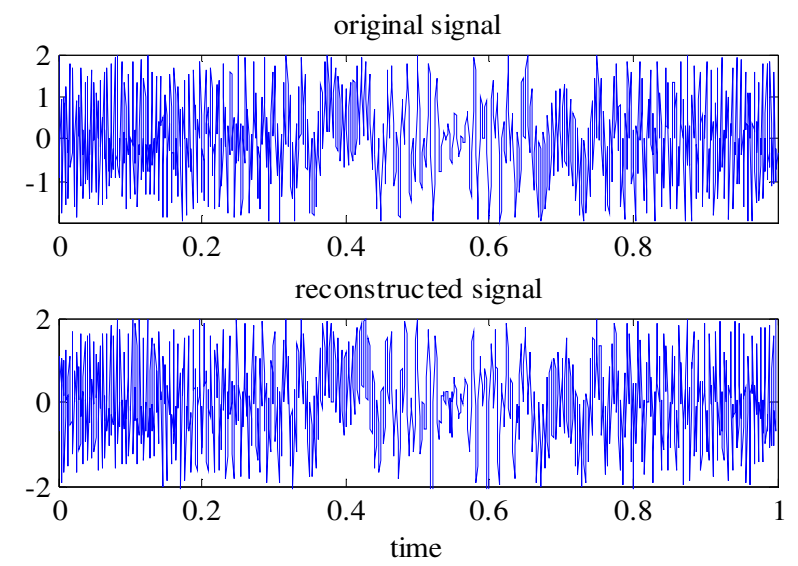

Figure 4. Original and reconstructed isolated LFM components

The DFT of original LFM components is shown in Fig.3 (top), while the DFT of noisy LFM signal with disturbances in shown in Fig. 3 (middle). The reconstructed DFT (after remodulation), with preserved amplitude and phase, is shown in Fig. 3 (bottom). We might observe that despite the strong disturbances that appeared in the corrupted signal in Fig. 3 (middle), the reconstructed signal (Fig. 3, bottom) is very close to the original one. Moreover, the original time domain LFM signals and their reconstructed version are shown in Fig. 4. In order to evaluate the precision of reconstruction, the mean square error (MSE) and mean absolute error (MAE) are calculated for original and reconstructed signal. The values of errors: $\mathrm{MSE}=0.0047$, while $\mathrm{MAE}=0.05$ (which is $2.5 \%$ of signal amplitude), prove high reconstruction accuracy.

\section{CONCLUSION}

A procedure for recovering the LFM signals from strong pulses and overlapping disturbances was addressed. The portions of desired signal components with no noise influence are selected using the L-estimation approach applied to LPFT, which is used to stationarize the LFM signal toward sinusoid. Dealing with a set of significantly reduced observations in the
TF domain, the linear relationship is established with the Fourier domain of sparsity, and the problem is cast as compressive sensing aiming at the recovery of narrowband signals in interference using $\ell_{1}$ reconstruction algorithm. The experimental evaluation of the proposed algorithm show high reconstruction accuracy for multicomponent LFM signals, providing low MSE and MAE between original non-noisy and reconstructed (de-noised) signal.

\section{ACKNOWLEDGMENT}

This work has been supported by the project CS-ICT (New ICT Compressive sensing based trends applied to: multimedia, biomedicine and communications), funded by Montenegrin Ministry of Science.

\section{REFERENCES}

[1] V. C. Chen: "Micro-Doppler effect in radar: Part I: Phenomenon, physics, mathematics, and simulation study," IEEE Trans. AES, vol. 42, no. 1,2006 , pp.2-21.

[2] F. Totir and E. Radoi: "Superresolution algorithms for spatial extended scattering centers", Digital Signal Processing, vol. 19, no. 5, Sept. 2009, pp.780-792.

[3] M. Martorella: "Novel approach for ISAR image cross-range scaling," IEEE Trans. AES, vol. 44, no. 1, 2008, pp. 281-294.

[4] J. Jeong and W. Williams, "Time-varying filtering and signal synthesis," in Time-Frequency Signal Analysis: Methods and Applications, B. Boashash, Ed., Wiley Press, 1992, ch. 17, pp. 389-405.

[5] J. Lerga, V. Sucic and B. Boashash, "An Efficient Algorithm for Instantaneous Frequency Estimation of Nonstationary Multicomponent Signals in Low SNR," EURASIP J. on Adv. in Signal Processing, SP/725189, Jan 2011

[6] L. Stankovic, I. Orovic, S. Stankovic, M. Amin, "Robust TimeFrequency Analysis based on the L-estimation and Compressive Sensing", IEEE SP Letters, vol.20, No.5, pp.499-502, May 2013.

[7] P.J. Huber, Robust Statistics, John Wiley\&Sons Inc., 1981.

[8] L. Stankovic, M. Dakovic, T. Thayaparan, Time-frequency Signal Analysis with Applications, Artech House, 2013.

[9] N. Zaric, I. Orovic, S. Stankovic, "Robust Time-Frequency Distributions with Complex-lag Argument," EURASIP Journal on Advances in Signal Processing, Vol. 2010, Article ID 879874, 10 pages, 2010

[10] L. Stankovic, I. Orovic, S. Stankovic, M. Amin, "Compressive Sensing Based Separation of Non-Stationary and Stationary Signals Overlapping in Time-Frequency," IEEE Trans. on Sig. Proc., vol. 61, no. 18, pp. 4562-4572, 2013

[11] X. Li, G. Bi, S. Stankovic, A. M. Zoubir, "Local polynomial Fourier transform: A review on recent developments and applications," Signal Processing, Vol. 91, No. 6, pp. 1370-1393, 2011

[12] E. Candes, J. Romberg J, T. Tao T "Robust uncertainty principles: Exact signal reconstruction from highly incomplete frequency information," IEEE Trans. IT, vol.52, no.2, 2006, pp.489-509.

[13] D. Donoho, “Compressed sensing," IEEE Trans. IT, vol. 52, no.4, 2006, pp. 1289 - 1306.

[14] S. Stankovic, I. Orovic, E. Sejdic, Multimedia Signals and Systems, Springer, 2012.

[15] R. Baraniuk, "Compressive sensing," IEEE SP Magazine, vol. 24, no. 4, 2007, pp. 118-121.

[16] B. Jokanovic, M. Amin, S. Stankovic, "Instantaneous frequency and time-frequency signature estimation using compressive sensing," SPIE Defense, Security and Sensing, Baltimore, United States, 2013

[17] P. Flandrin, P. Borgnat, "Time-Frequency Energy Distributions Meet Compressed Sensing," IEEE Trans. SP, vol.58, 2010, pp.2974-2982.

[18] S. Stankovic, I. Orovic, M. Amin, "Compressed Sensing Based Robust Time-Frequency Representation for Signals in Heavy-Tailed Noise," Information Sciences, Signal Processing and their Applications, ISSPA 2012, Canada, 2012 